\title{
THE EAR AND AVIATION
}

\author{
EUGENE R. LEWIS, M.D. (Dubuque, Iowa) \\ Lieutenant-Colonel, M. C., U. S. Army \\ MINEOLA, L. I., N. Y.
}

In undertaking to train a man to learn to fly, the Army demands that he be a normal man in good health. Therefore, each applicant for this training is given a careful complete physical examination, including the special senses. The ear examination includes tests of soundperception, and of the motion-perception of the vestibular apparatus; the regulation requirements demand normal acuity of perception.

The difference between the man on the ground and the man in the air lies in the fact that the former can stand still, the latter cannot. When the flier walks across the field to his plane, all his motor coordinations are concerned with maintaining the proper relation between his body and the element which is supporting its weight - the earth. When he straps himself in the seat before flight, he practically straps wings to his body, thenceforth, until the end of his flight, every motor coordination is concerned with maintaining a proper relation with the new element which is supporting his weight - the air. The only means he possesses of adjusting his relation with the new weightsupporting element is the plane; while flying, all motor coordinations, whether carefully calculated or instinctively performed, are concerned exclusively with controlling the plane. The promptness and efficiency with which motor coordinations are performed depend directly on the acuteness of sensory perceptions.

Rising in the air in an aeroplane is made possible only by rapid motion. Acuity of motion-perception assumes much greater importance to the flier than to the pedestrian, and in order to appreciate the full importance of this, one must have a clear conception of the component senses going to make up motion-perception. Muscle-and-joint sense, splanchnic or visceral sense, kinesthetic sense - all grouped for convenience under the term "deep sensibility," vestibular sense, vision and tactile sense each participate in the composite of general motion-perception.

\section{THE IMPORTANCE OF MOTION-PERCEPTION TO THE AVIATOR}

Deep sensibility, on the ground, is practically exclusively concerned with sensing the effect of the pull of gravity on the body; in the air it is also concerned with sensing the effect on the body of two other pulls, that of the plane's propeller, and that of centrifugal force on 
curves. Impulses generated by these three pulls coming in via the deep sensibility tract must undergo accurate analysis in the brain and be properly estimated and labeled if confusion and misinterpretation are to be avoided in the sensorium. While such analysis is accomplished by normal individuals, it is only at the exclusion of a certain amount of the more accurate sensing of the pull of gravity. Whereas, on the ground practically 100 per cent. of this incoming information expresses gravity pull, a less percentage of gravity pull is expressed by it in the air.

Vision, possibly the most important of all motion-perceiving senses on the ground, suffers some impairment of its usefulness in the air by reason of the reduction in the number of visible elements in the new environment such as the usual objects making up the landscape. When darkness or cloud further reduces the utility of vision, this sense becomes almost eliminated as a source of guiding information to the flier.

Tactile sense contributes less than any of the other three senses to motion-perception on the ground; to the flier, although insulated by warm clothing and helmet, it is still of value as a source of guiding information.

Vestibular sense suffers no depreciation in utility in the air as compared with on the ground. Its sole function has always been, and continues unaltered in any way to be, pure sensing of motion. In flying, therefore, its function assumes a relatively greater importance than that of the other special senses cooperating with it to furnish the individual with his composite of knowledge concerning motion.

\section{VESTIBULAR TESTS}

The motion-perceiving apparatus of the internal ear is subjected to stimulation by motion of certain standard quantity and quality, and the results are observed according to uniform standard methods. Two results are noted-a sensory result, the subjective sensation of motion, and a motor result, involuntary movement of the eyes. When the subjective sensation of motion is in accord with fact, we call it normal sensing of motion; when it is not in accord with fact, we call it "vertigo." The only difference between normal motion-perception and vertigo lies in the sensing of motion being in accord with, or contrary to fact. The most practical means of applying motion stimulus is by the rotating chair, since the application of motion in a linear direction, for the period of time and in the intensity necessary to elicit certain standard responses to that stimulus would necessitate apparatus entirely too bulky to be susceptible of practical application under ordinary conditions of office examination. By making use of a rotational motion stimulus instead of a linear motion stimulus, it was 
possible to work out a standard means of applying motion stimulus in certain definite quality and quantity in a manner, and by means of an apparatus easily handled in an office. For this reason only, the subject of the tests of the vestibular apparatus is made to experience rotational vertigo. An additional advantage in using the rotating chair is that it applies motion stimulus of a character to produce a more enduring stimulation of the end organs of the semicircular canals.

Motion in a linear direction applied to a fluid contained in a closed semicircular canal is physically incapable of setting up an enduring flow of that fluid, just as rotational motion applied to a fluid contained in a straight canal cannot set up an enduring flow.

\section{NYSTAGMUS PRODUCED BY MOTION STIMULATION}

Ewald's experiment long ago determined that involuntary pulling of the eyes in a certain definite direction and plane occurs during the time the fluid in a normal semicircular canal is made to flow in one direction; and that during the time this fluid is made to flow in the opposite direction involuntary pulling of the eyes in the opposite direction occurs. By applying rotational motion, it is possible to reproduce Ewald's experiment in effect, as a test of eye-reactions to vestibular stimulation; and when the character and intensity of rotational stimulus is standardized, comparisons of the results can be made and a normal eye-reaction determined. This motor expression of motion stimulation is nystagmus.

\section{MEASURING VERTIGO}

The normal man experiences a sensation of vertigo for between fifteen and thirty seconds after being turned according to standard technic. Evidence of this subjective sensation may be had by voluntary or involuntary testimony; voluntary testimony, such as "I'm turning to the right," "I'm still turning to the right," etc., during the persistence of the subjective sensation; involuntary testimony, such as pointing test and falling. Standard tests make use of involuntary testimony in all cases; occasionally this is amplified by voluntary testimony with advantage. In observing the pointing before turning a very important element in the test can be injected by implanting in the mind of the applicant the definite idea that he is to attempt to determine the location in space of the observer's finger solely by registering in his memory the location of it according to his tactile sense. This can be augmented by having him touch the observer's finger in more than one position (pointing test) ; as, for instance, directly in front of the right hand, come back and touch; then locate again 30 degrees outward and come back and touch; the same procedure in front of the left hand. This implants in his mind the fundamental idea of 
being able to orientate himself solely by means of afferent impulses from his tactile end-organs. After standard rotation to the right, for example, normal man experiences certain very definite vertigo, a subjective sensation of turning to the left in the same plane as the rotation for a normal period of time. If the pointing test is carried out during this period of vertigo, instead of succeeding in pointing accurately to the testing finger he executes the pointing in accordance with his subjective sensation of motion. Feeling that he is turning definitely away from the testing finger to the left, he reaches for it to the right. This is normal past-pointing.

METHOD OF APPLYING THE PAST-POINTING TEST

The insulation of the applicant during this test should be as perfect as possible. A black domino mask should be used, absolute quiet should be maintained, olfactory impressions should be shunted out, and he should be left as solely as possible dependent on the information brought to him along the vestibular tract alone. The applicant should be definitely instructed before turning that he should not expect a verbal order to touch the observer's finger, raise his hand and come back, and attempt to find it after the turning; he should be practiced before turning in executing his touch, raising the hand, and coming back to find the finger on receipt of the signal from the observer's finger as it comes into the position which it maintains during the test - the observer bringing up his finger into position so as to tap the applicant's finger as a signal for him to execute his pointing without verbal command. It is very important for the applicant's finger to find a finger of the observer when he comes down in search of the finger which is testing him. Otherwise, there is injected into his mind a disconcerting element of dissatisfaction in having failed to find the finger for which he was searching. For this purpose the index finger of the observer's left hand can be held in readiness to furnish the touch necessary to shunt out this sense of failure. In observing the past-pointing after rotation the observer's right index finger should be definitely fixed against the observer's hip, so that visual attention to it on the part of the observer can be dispensed with, the hip rest insuring its remaining definitely where it was when the applicant first touched it in making the pointing test. The observer's eyes can then be free to watch the applicant's finger at the top of the swing. Pastpointing at the top of the swing is just as definitely normal pastpointing as at the completion of return to touch. Many cases compensate after evincing a normal tendency, let us say, to past-point outward with the right hand when they should do so, and subsequently execute a compensatory touch or inward-pointing at the bot- 
tom of the return. In such cases the pointing should be registered as that executed at the top of the swing, which is the primary and clean response before it has been altered by the subconscious or conscious compensation effected by other mental processes. Visual attention on the part of the observer to the applicant's hand at the beginning of his downward pointing is of enormous importance, and it should be very carefully observed as part of the standard technic.

\section{APPLICATION OF THE FALL TEST}

The fall test is similar. A normal man, on attempting to sit upright after leaning forward during right rotation, feels that he is turning to the left, for instance, and so gives involuntary expression to this sensation by falling to the right on attempting to assume an erect sitting posture.

These tests can be completed in less than four minutes. The neurologist will especially appreciate the extent to which the central nervous system has been examined, and determined to be normal during these tests of the vestibular apparatus.

\section{COMMENT}

In view of the foregoing, it is apparent that in flying, motion takes on a much greater importance as regards potential safety or disaster for the individual than it possesses on the ground, and that motionperception is commensurately of greater importance in the air than on the ground.

Regardless of the actual percentages which would express the shares of vision, deep-sensibility, and vestibular and tactile sense in the total of motion-sensing on the ground, it is established that three of these four are reduced in efficiency by conditions incidental to flying, and the fourth, vestibular sense, is not so reduced, and is therefore of relatively increased importance. It follows that it is of prime importance to determine that men to be trained as fliers possess normal vestibular apparatus. So important is it for the flier to possess normal vestibular acuity of motion-perception that no man should be permitted to begin training as a pilot who has not definitely shown normal reactions to vestibular tests.

This, however, does not end the otologist's responsibility in aviation. It must be borne in mind that physical deteriorations of the vestibular apparatus are always possibilities. Cases have been encountered in which men have gone into the service possessed of normal vestibular sense, and subsequently developed marked impairment of their vestibular function, seriously reducing their flying ability. Reexamination of all fliers at intervals is just as necessary to proper maintenance of the flying service as is the first examination of applicants for admission to this service. 